\title{
WILEY-VCH
}

\section{Effect of Core Liquid Surface Tension on The Liquid Marble Shell}

Pradip Singha, Nhat-Khuong Nguyen, Kamalalayam Rajan Sreejith, Hongjie An, Nam-Trung Nguyen, * Chin Hong Ooi*

Pradip Singha, Nhat-Khuong Nguyen, Dr. K R Sreejith, Dr. Hongjie An, Prof. Nam-Trung Nguyen, Dr. Chin Hong Ooi Queensland Micro- and Nanotechnology Centre, Griffith University, 170 Kessels Road,

Nathan 4111 Queensland, Australia

E-mail: nam-trung.nguyen@griffith.edu.au, c.ooi@griffith.edu.au

Keywords: liquid marble, surface tension, interfacial jamming

Liquid marble is a non-wetting droplet coated with micro- or nanometer sized particles and exhibits great versatility as a standalone system. Unique features such as low evaporation rate, low friction, and a porous shell enable it to be a potential tool for gas sensing, cell culture and drug delivery. With the growing number of interests in these fields, it is important to understand the structure of the liquid marble shell and how it behaves with the variation of the surface tension of the core liquid. This paper investigates the thickness and the mass of liquid marble shell at various surface tension values. Surfactant mediated surface tension reduction allows the encapsulating particles to penetrate more into the core liquid and decreased the shell thickness. The shell thickness decreases significantly with the surface tension, whereas the mass remains almost constant. Interestingly, the trend reverses with further decrease of the surface tension. In this regime, both the thickness and the mass of the shell increases due to interfacial jamming. This work provides a new insight into understanding the contribution of the particle distribution at the shell for the stability and the measurement of the effective surface tension of a liquid marble.

\section{Introduction}

A liquid marble is a non-wetting droplet encapsulated by micro- or nanometer sized hydrophobic particles. ${ }^{[1]}$ Liquid marble attracts increasing attention in microfluidics due to its 


\section{WILEY-VCH}

flexible manipulation, ${ }^{[2]}$ high stability, ${ }^{[3-7]}$ well controlled evaporation rate, ${ }^{[8-11]}$ and insusceptibility to contamination. ${ }^{[2,12,13]}$ These advantages make the liquid marble an important platform for microfluidic applications. ${ }^{[13-15]}$ A liquid marble comprises a core liquid and a particulate shell. Liquid marble manipulation schemes tend to exploit properties of the encapsulating particles. The encapsulating particles determine the elasticity, ${ }^{[16]}$ robustness, ${ }^{[4]}$ and the effective surface tension of a liquid marble. ${ }^{[17,18]}$ Furthermore, liquid marbles with functional shells can be manipulated using light, ${ }^{[19-22]}$ temperature gradient, ${ }^{[23]}$ surface tension gradient, ${ }^{[24,25]}$ magnetic field, ${ }^{[26-29]}$ electric field, ${ }^{[30-34]}$ mechanical force, ${ }^{[4,35]}$ and $\mathrm{pH} \cdot{ }^{[36-38]}$ The porous liquid marble shell enables gas exchange, which allows it to serve as a gas sensor, ${ }^{[39,40]}$ a microreactor for gas-phase reactions, ${ }^{[41,42]}$ a carbon dioxide capturing tool, ${ }^{[43]}$ as well as a cell culture platform. ${ }^{[15,44,45]}$ The behavior and the structure of a liquid marble shell depends on the preparation method, morphology of the encapsulating particles, and the properties of the core liquid. This paper investigates the relationship between the core liquid and the properties of the shell.

A liquid marble shell represents a flexible superhydrophobic surface analogous to CassieBaxter wetting. ${ }^{[46]}$ The shell consists of encapsulating particles and pores that prevent direct contact between the core liquid and the carrying substrate. There are only a few works in available literature that show the structure of a liquid marble shell. ${ }^{[12,47,48]}$ Notable are the observations by Aussillous et al.,. ${ }^{[12]}$ and Bormashenko et al. ${ }^{[48]}$ using an optical microscope and environmental scanning electron microscope (ESEM), respectively. Both studies revealed the existence of interparticle gaps at the surface of a liquid marble. Lin et al. investigated the shell of a liquid marble with an optical microscope ${ }^{[49]}$ The team reported that a liquid marble shell is not fully covered with particles. Furthermore, the pores on the shell causes liquid marbles to evaporate. ${ }^{[50]}$ Nguyen et al. investigated on the shell structure using a confocal microscope. ${ }^{[47]}$ The team showed that fine particles are packed in between coarse particles within the shell. The particles at the innermost layer of the shell always penetrate the core liquid. ${ }^{[47]}$ Wang et al. 


\section{WILEY-VCH}

reported that the extent of penetration depends on the surface tension of the core liquid. ${ }^{[51]} \mathrm{A}$ reduction in the surface tension of the core liquid increased the penetration into it and decreased the lifetime. Prior works discuss only on the structure of the shell. There has yet to be a detailed study on the properties of the shell in response to the core liquid surface tension.

The integrity of a liquid marble depends on the properties of the shell. Therefore, it is essential to study the behavior of the shell to understand its effects on both the static and the dynamic properties of a liquid marble. Our present work investigates the effect of the surface tension of the core liquid on the shell. The surface tension was controlled using known quantities of surfactant. Shell thickness decreased with the surface tension up to a threshold. Decreasing shell thickness was caused by a higher degree of penetration of the particles into the droplet. Further decrease caused interfacial jamming of the particles which resulted in increasing shell thickness. The results of this work could be useful to understand the influence of the shell on the stability of a liquid marble and to address the inconsistencies of the measurement of the effective surface tension of a liquid marble.

\section{Theory}

A liquid marble consists of a core liquid droplet and encapsulating particles. Encapsulating particles are adsorbed onto the core liquid surface during the formation process of the liquid marble. Some particles of the innermost layer of the liquid marble always penetrate the core liquid after attachment, Figure 1. ${ }^{[47]}$ The attachment of the particles releases a relatively large amount of energy. Therefore, a relatively large detachment energy $(\Delta G)$ is required for the particles to completely submerge into the liquid: ${ }^{[12,13]}$

$\Delta G=\pi R_{P}^{2} \gamma_{l a}(1-\cos \theta)^{2}$

where $R_{\mathrm{p}}$ is the radius of the particle, $\gamma_{l a}$ is the liquid-air interfacial tension, and $\theta$ is the threephase contact angle. The decrease in the core liquid surface tension $\gamma_{l a}$ reduces the energy required for the particles to detach from the droplet surface and immerse fully into the core 


\section{WILEY-VCH}

liquid. Therefore, we hypothesize that the particles immerse more into the core liquid, which directly reduces the thickness of the encapsulating layer. We assume that the variation in the thickness is independent of the liquid volume.

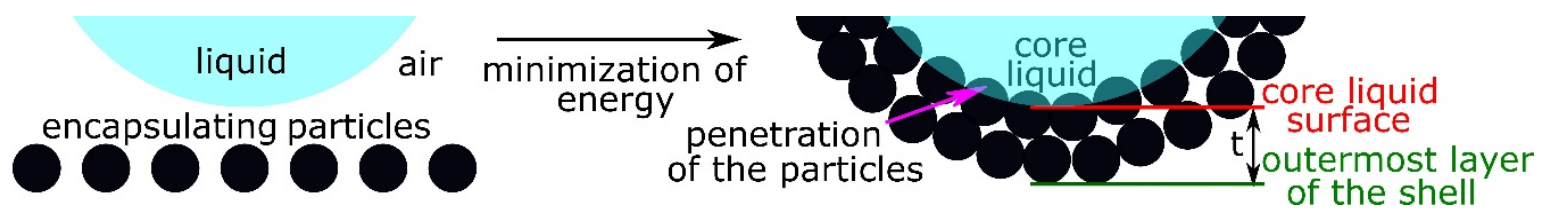

Figure 1. Schematic of the penetration of the encapsulating particles during fabrication, $t$ represents the shell thickness.

A decreasing surface tension also reduces the inward pulling force on the liquid surface molecules, diminishing the ability of the liquid surface to oppose deformation. At the same time, more surfactant molecules are adsorbed onto the solid-liquid interface which increases the hydrophilicity of the particles. ${ }^{[11]}$ The transition in the wetting behavior of the particles caused a capillary rise between the particles into the liquid surface due to the positive Laplace pressure, ${ }^{[51-55]}$ Figure 2a and Figure 2b. Consequently, the liquid spacing between the particles at the innermost layer increases, Figure $2 \mathrm{~d}$. The liquid column rise between the particles tends to push the particles away from each other, ${ }^{[51]}$ resulting in sparse packing at the droplet surface. Therefore, we hypothesize that the amount of particles required to encapsulate the surface area of a droplet decreases with surface tension.

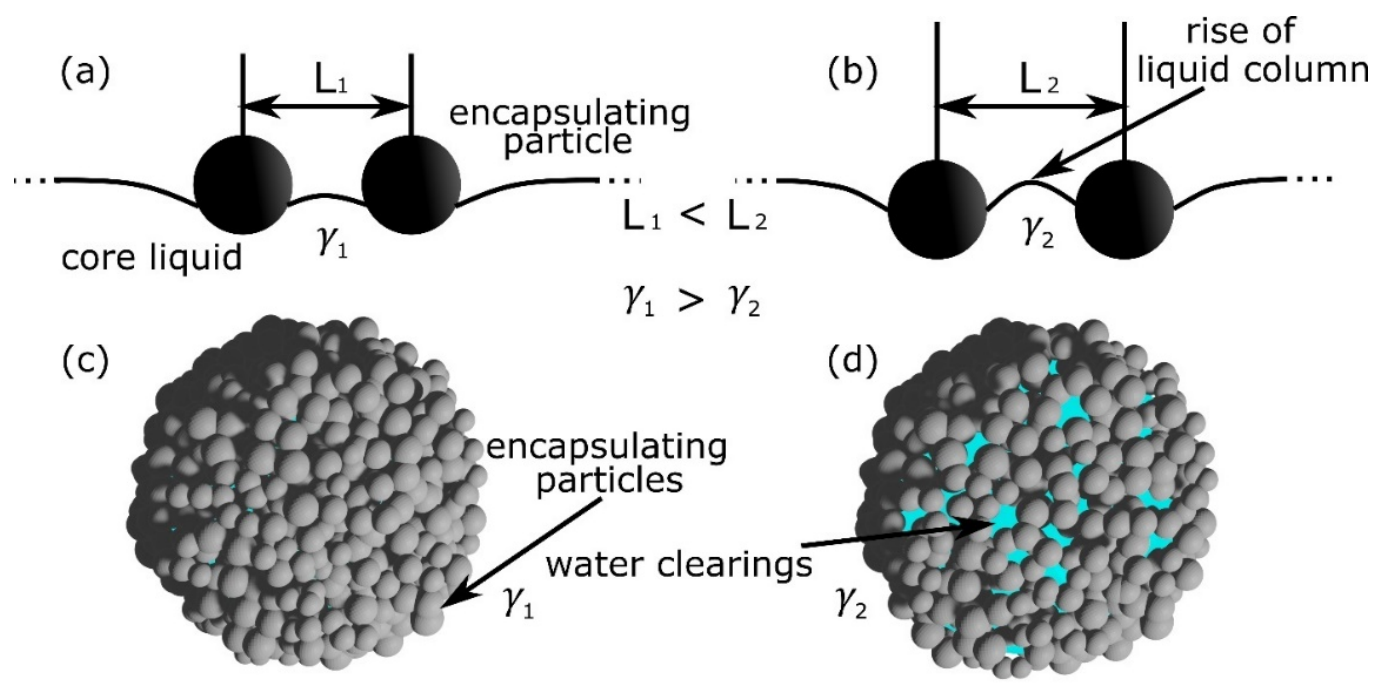




\section{WILEY-VCH}

Figure 2. Schematic of the meniscus between two encapsulating particles a) at higher surface tension, and b) at lower surface tension. Depicted particles represent the innermost layer. Schematic three-dimensional illustration of liquid marbles at c) higher surface tension, and d) at lower surface tension. $L$ and $\gamma$ represents the interparticle distance and the surface tension of the core liquid respectively.

In addition, surface tension reduction flattens the droplet. ${ }^{[56]}$ As a result, the droplet surface area increases. Hence, we expect that more particles are needed to encapsulate the droplet surface. Both hypotheses for the amount of encapsulating particles of a liquid marble have competing effects. In our study, we investigate the mass of the encapsulating particles to test these competing hypotheses.

\section{Experimental Procedures}

A $30-\mu 1$ deionized (DI) water droplet was dispensed onto the lycopodium powder bed placed over a microplate vortex mixer (RATEK). The droplet was rolled over the powder bed for 33.5s at a mixer revolution speed of $145 \mathrm{rpm}$, Figure 3. This fabrication method was used to prepare all the liquid marbles to keep the rolling parameter constant. Lycopodium powder (Sigma Aldrich) with an average diameter of $27 \pm 3 \mu \mathrm{m}$ was used as encapsulating particles., Figure 4a.

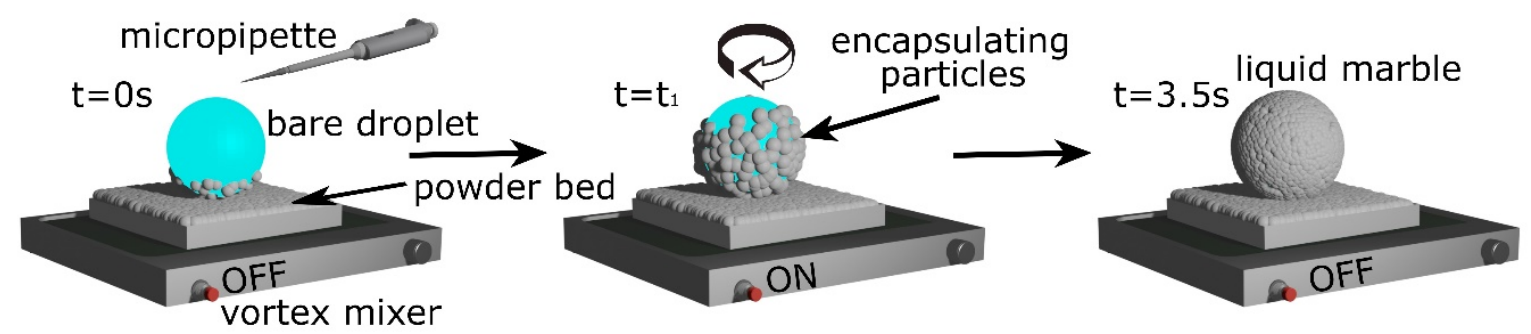

Figure 3. Formation of liquid marbles using a vortex mixer. 


\section{WILEY-VCH}

To reduce the surface tension of the core liquid, aqueous sodium dodecyl sulfonate (SDS) was added to the droplet. The surface tension of SDS solution was characterized using the pendant drop method and the Drop Analysis plugin in ImageJ, ${ }^{[57]}$ Figure $4 \mathrm{~b}$.
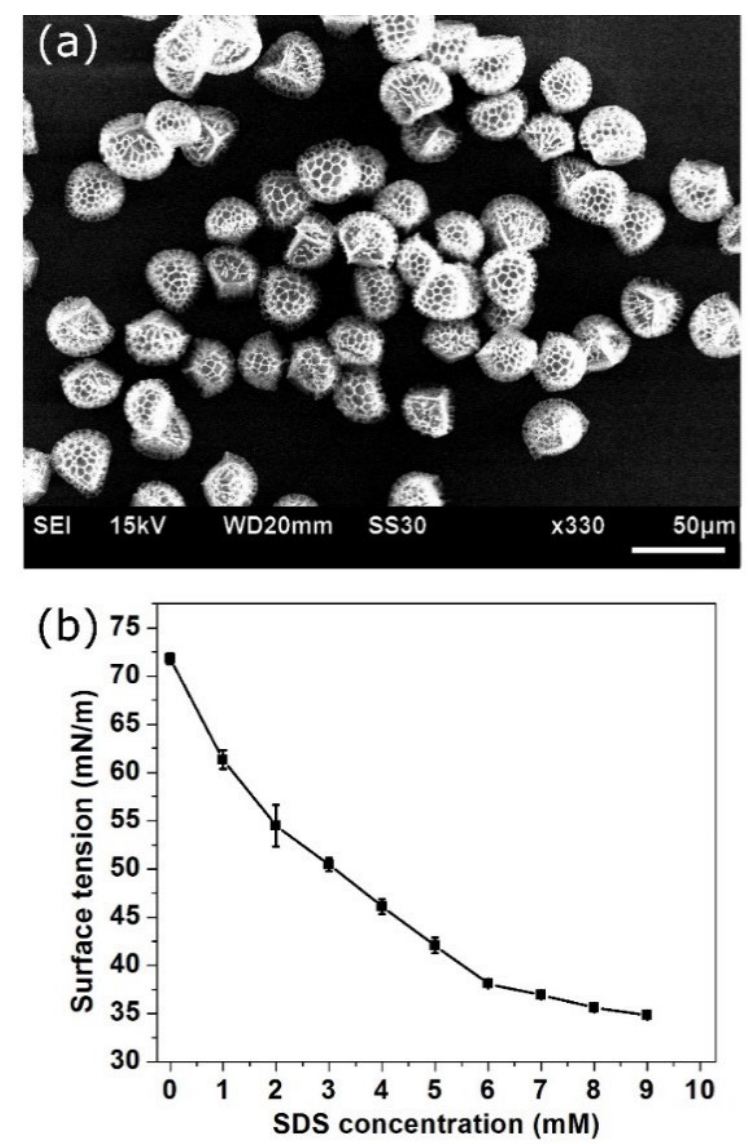

Figure 4. a) Scanning Electron Microscope image of lycopodium powder, b) Surface tension of aqueous SDS solution at $22^{\circ} \mathrm{C}$.

Prepared liquid marbles containing DI water and SDS solution were gently rolled on a clean solid substrate to eliminate excess coating powder. The bottom view of the liquid marble was observed using an inverted microscope. First, we focused on the outermost layer of the shell as shown with the green line in Figure 1. Then, we changed the focus to the core liquid surface as shown with the red line in Figure 1. The elevation between the outermost layer and the core liquid surface is the measurement of thickness in our study. The mass, $m_{p}$ of the encapsulating particles were measured as:

$m_{p}=m_{m}-m_{l}$ 


\section{WILEY-VCH}

where $m_{m}$ is the mass of the liquid marble, $m l$ is the mass of the core liquid.

\section{Results and Discussions}

A 30- $\mu 1$ DI water liquid marble had a shell thickness of $64 \pm 2 \mu \mathrm{m}$. To test our assumption that the shell thickness is independent of volume, we performed the experiment with DI water liquid marble of volumes ranging from $25 \mu 1$ to $50 \mu$. The measured thicknesses did not change significantly across different volumes, Figure 5a. Thus, we neglected the volumetric effects, and only 30- $\mu 1$ liquid marbles were used for subsequent discussions. We measured the shell thickness at a minimum surface tension of $43 \mathrm{mN} / \mathrm{m}$. Liquid marbles in this surface tension collapsed within $70-120$ s after transferring to the glass slide on the microscope. Further decrease of the surface tension would result in an unstable liquid marble.

The shell thickness decreased significantly from $64 \pm 2 \mu \mathrm{m}$ to $52 \pm 2 \mu \mathrm{m}$ as the surface tension decreased from $72 \mathrm{mN} / \mathrm{m}$ to $54 \mathrm{mN} / \mathrm{m}$, Figure 5 b. Interestingly, further decrease of the surface tension resulted in a rapid increase of the shell thickness. The thickness increased to 77 $\pm 2 \mu \mathrm{m}$ at the surface tension of $43 \mathrm{mN} / \mathrm{m}$. We therefore split the discussion into two regimes:

regime I, where the thickness decreased; and regime II, where the thickness increased with decreasing core liquid surface tension. 

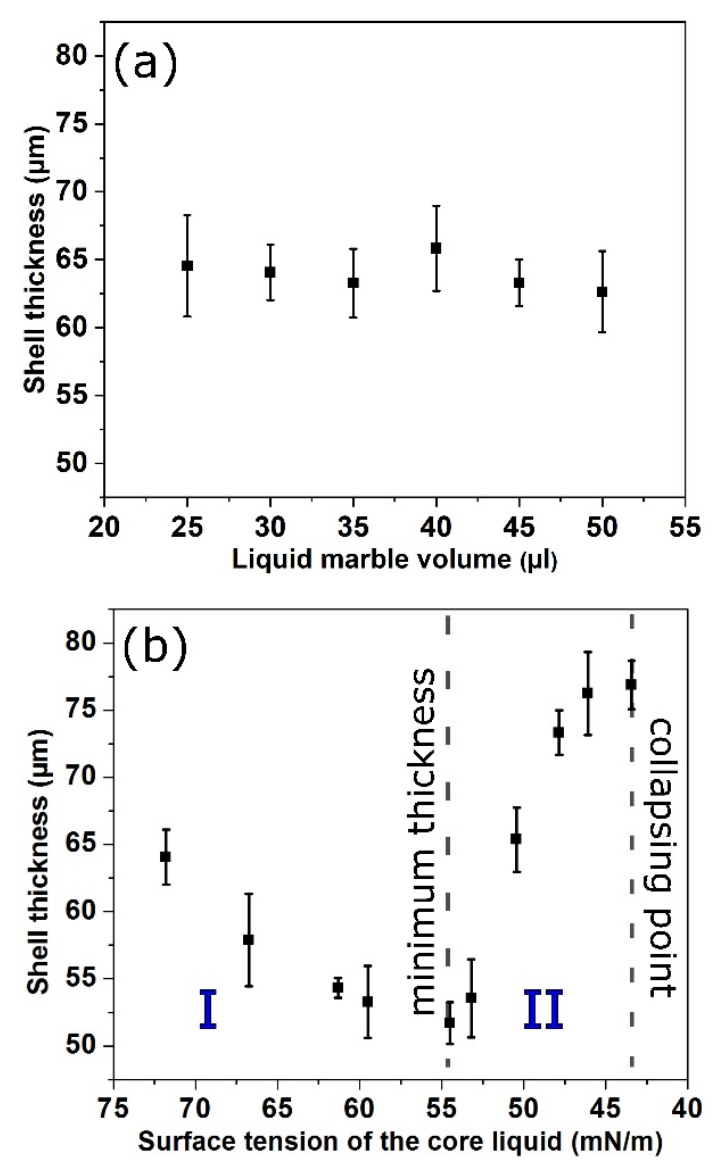

Figure 5. Shell thickness of a) a water-based liquid marble with volumes of $25 \mu 1$ to $50 \mu 1$, and b) a $30-\mu 1$ liquid marble at various surface tension.

In regime I, the decrease in shell thickness can be attributed to the combined effect of the penetration of the lycopodium into the core liquid and the enhancement of the hydrophilicity of lycopodium with the decrease of the surface tension. To demonstrate that the hydrophilicity of the lycopodium powders would enhance with the reduction of surface tension, we determined the contact angle of a bare droplet as a function of surfactant concentration, resting on a lycopodium powder bed.

Figure 6a shows the contact angle of a bare droplet with varying concentration of SDS resting on a lycopodium powder bed. The contact angle of a DI water droplet is $134^{\circ}$ and was reduced to $123^{\circ}$ when the surfactant concentration is $1 \mathrm{mM}$ (surface tension of $61 \mathrm{mN} / \mathrm{m}$ ). Further decrease of the surface tension did not affect the contact angle significantly. This observation can be attributed to the petal effect exhibited by the lycopodium powder. 


\section{WILEY-VCH}

Lycopodium-based surfaces manifest Cassie-impregnated wetting state. ${ }^{[58]}$ In such case, the droplet is characterized by high adhesion and high hysteresis of the apparent contact angle. When the SDS molecules were introduced into the core liquid, a fraction of it adsorbed on the lycopodium surfaces via van der Waals forces between the alkyl chain of the surfactant and hydrophobic groups on the particle. ${ }^{[59]}$ Thus, a monolayer was formed which exposed the charged surfactant head groups to the aqueous phase. According to Lucassen-Reynders equation, the decrease of the surface tension results in more adsorption of the surfactant molecules at the solid-liquid interface and increases the hydrophilicity. ${ }^{[60]}$ We speculate that the adsorption of the surfactant molecules to the lycopodium surface enhanced the adhesiveness with the core liquid, which caused the encapsulating particles to penetrate more into the core liquid and reduced the shell thickness.
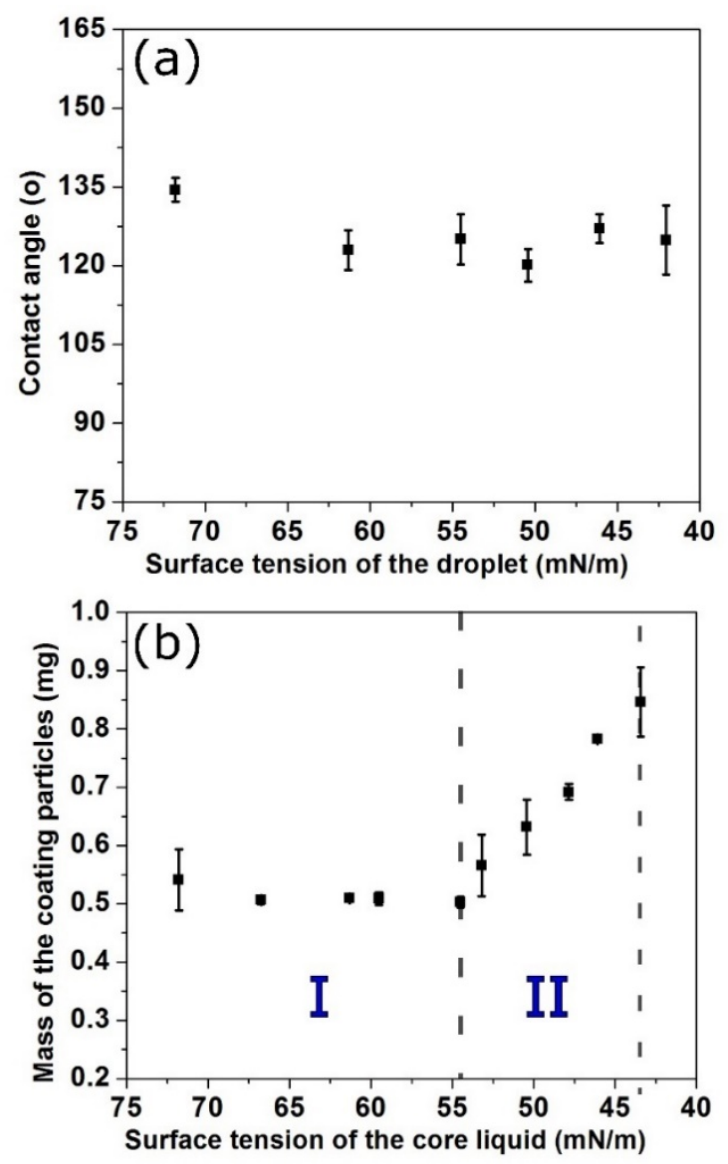

Figure 6. a) SDS concentration versus contact angle, and b) mass of the encapsulating particles of a $30-\mu 1$ liquid marble at various surface tension. 


\section{WILEY-VCH}

We measured the mass of the encapsulating particles in regime I. In our study, the mass of the encapsulating particles was $0.54 \pm 0.05 \mathrm{mg}$ at a surface tension of $72 \mathrm{mN} / \mathrm{m}$ and remained nearly constant with further reduction of the surface tension up to $54 \mathrm{mN} / \mathrm{m}$, Figure $6 \mathrm{~b}$. The competing effects of larger interparticle distance and larger surface area seemed to cancel out each other at this regime.

In regime II, the shell thickness increased with the decrease of surface tension. This increase is attributed to the interfacial jamming of lycopodium powder at the droplet surface during the fabrication. The shape of the droplet under the action of vortex mixer is balanced by the surface energy and the kinetic energy, generated through the vortex mixer. ${ }^{[61]}$ We know that the surface energy reduces with the surface tension. Below the threshold surface tension of $54 \mathrm{mN} / \mathrm{m}$, kinetic energy seemed to dominate surface energy. As a result, the droplet deformed from its original shape under the action of the vortex mixer. The deformed surface collected a large amount of lycopodium through the enlarged solid-liquid interfacial area, Figure 7. Continual reduction of the surface tension deformed the droplet more and collected a larger amount of encapsulating particles during the fabrication process. Therefore, the mass of the encapsulating particles increased in regime II, Figure $6 \mathrm{~b}$, which reached $0.84 \pm 0.06 \mathrm{mg}$ at a surface tension of $43 \mathrm{mN} / \mathrm{m}$. Once the rotation stopped, the surface tension force retracted the surface area and reduced the interfacial area. The retraction of the surface increased the interfacial particle concentration. When the specific interfacial area became comparable to that of close packing of particles, interfacial jamming occurred ${ }^{[62,63]}$ and surface area retraction was arrested. ${ }^{[64]}$ Interfacial jamming retained morphological coarsening and overlapping of the particles at the liquid-air interface. Therefore, it stabilized two phase morphologies with irregular interfacial shapes. So, the effect of sparse packing with the decrease of surface tension is not significant in this regime. Conversely, the effect of collection of particles through larger droplet contact area at lower surface tension is dominating in this regime. In our experiment, the profile of the liquid marble at low surface tension became irregular and did not fit the theoretical droplet 


\section{WILEY-VCH}

curve in Low-Bond Axisymmetric Drop Shape Analysis (LB-ADSA), ${ }^{[48,65]}$ Figure 8b, which proved the presence of interfacial jamming effect. Another possible reason for interfacial jamming could be due to the increase in droplet contact area with the substrate. ${ }^{[64,66]}$ Contact radius of the droplet with the substrate is calculated with Equation $3:^{[1,12]}$

$l / k^{-1}=(2 / 3)^{\frac{1}{2}}\left(R_{0} / k^{-1}\right)^{n}$

where, $l$ is the contact radius, $k^{1}$ is the capillary length of the droplet, $R_{0}$ is the radius of the droplet, $n=3 / 2$ for puddles (where, $\mathrm{R}_{0}>>\mathrm{k}^{-1}$ ), $n=2$ for smaller droplets (where $\mathrm{R}_{0}<<\mathrm{k}^{-1}$ ).

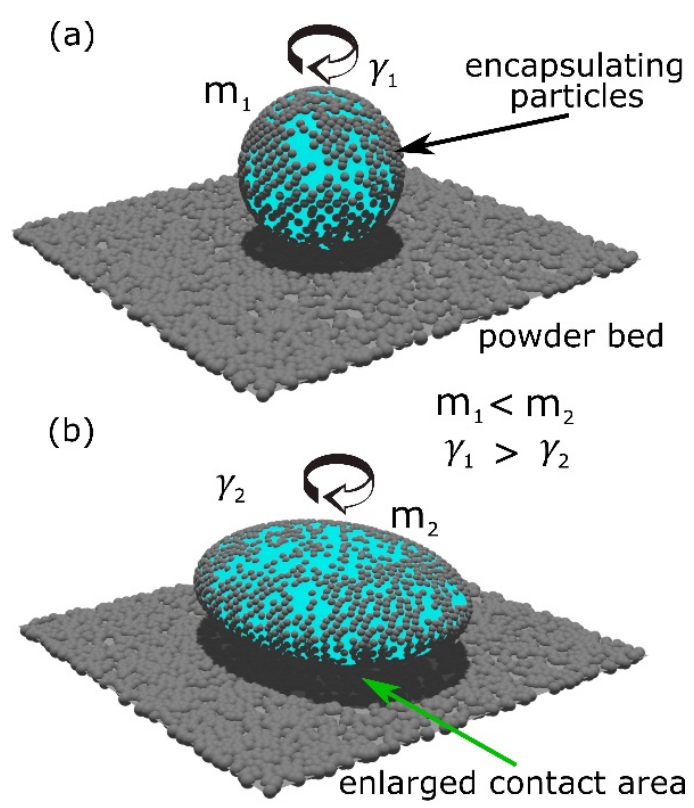

Figure 7. Illustration of the collection of the encapsulating particles through the contact area of a droplet with a) a higher surface tension, and b) a lower surface tension, under the action of vortex mixer, $m$ and $\gamma$ represents the mass of the coating and the surface tension of the core liquid respectively.

According to Equation (3), the contact area increases with the decreasing surface tension. This process increases the friction between the droplet and the substrate, hence reducing the mobility, which in turn affects the interfacial jamming effect. ${ }^{[64]}$ Therefore, interfacial jamming effect is more favourable with the decrease of the surface tension, which agrees well with Geyer et al. ${ }^{[66]}$ In our study, the overlapping of the particles due to the interfacial jamming increased 


\section{WILEY-VCH}

the shell thickness, regime II, Figure 5b. The thickness increased to $77 \pm 2 \mu \mathrm{m}$ at a surface tension of $43 \mathrm{mN} / \mathrm{m}$.
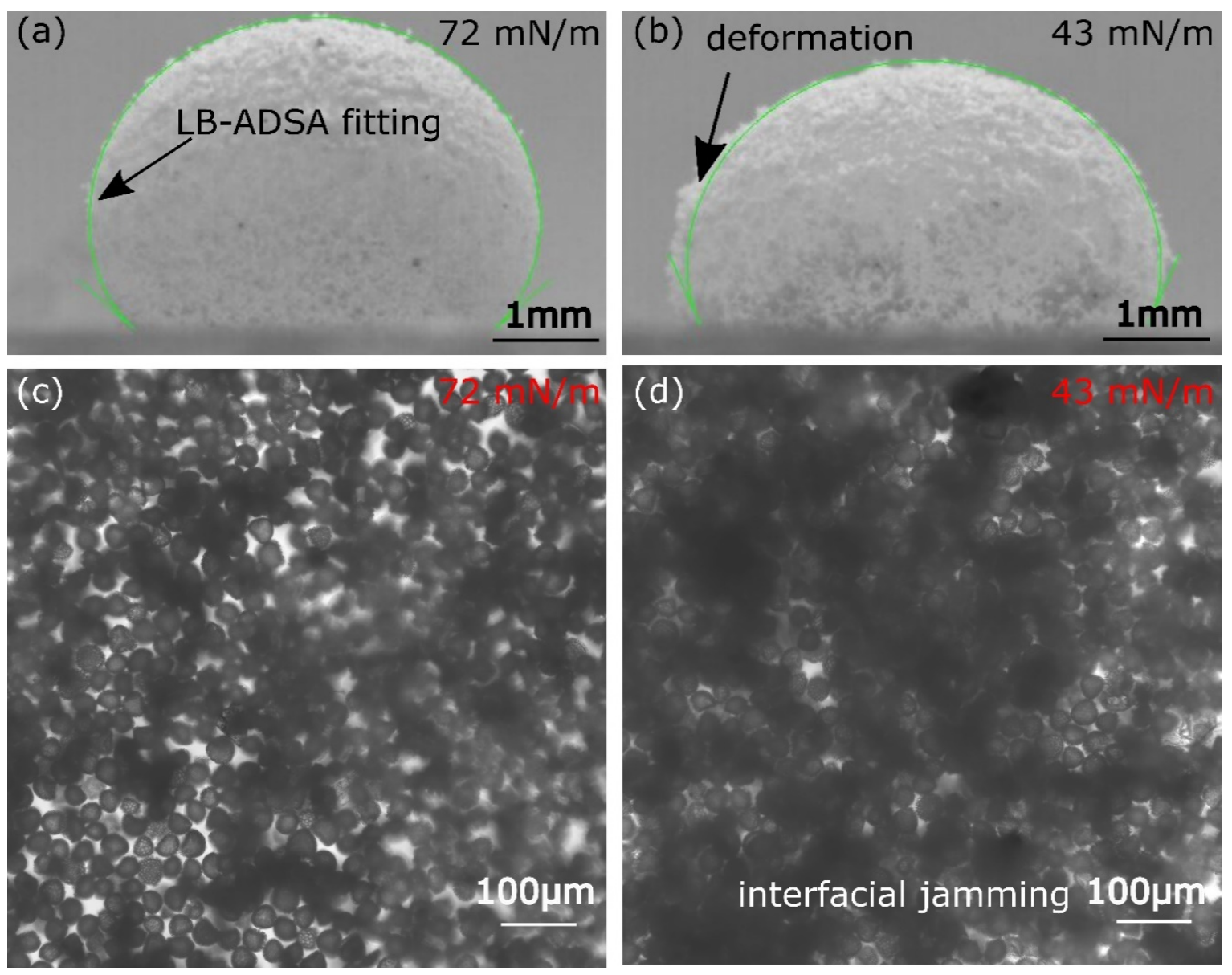

Figure 8. Side view of a liquid marble a) without interfacial jamming, b) with interfacial jamming. Microscopic view of the innermost layer of the liquid marble c) without interfacial jamming, d) with interfacial jamming.

Next, we demonstrated the sensitivity of the liquid marbles to ammonia vapor with different surface tensions. Phenolphthalein was added to the core liquid solution at a volume ratio of $0.5 \%$ for the liquid marbles, kept in ambient air. First, the prepared liquid marbles exhibited a yellow color due to the encapsulating lycopodium particles, Figure 9a. Then, a beaker containing ammonia solution was placed in the proximity of the liquid marbles to produce ammonia vapor. All the liquid marbles turned pink in the presence of ammonia vapor, Figure 


\section{WILEY-VCH}

9b. Pores in the liquid marble shell allowed the diffusion of ammonia vapor into the core liquid and resulted the color change. We were unable to differentiate the sensitivities to ammonia vapor across liquid marbles of different surface tensions as lycopodium powder is yellow and opaque. The shell impairs colorimetric analysis required to accurately differentiate the sensitivities.

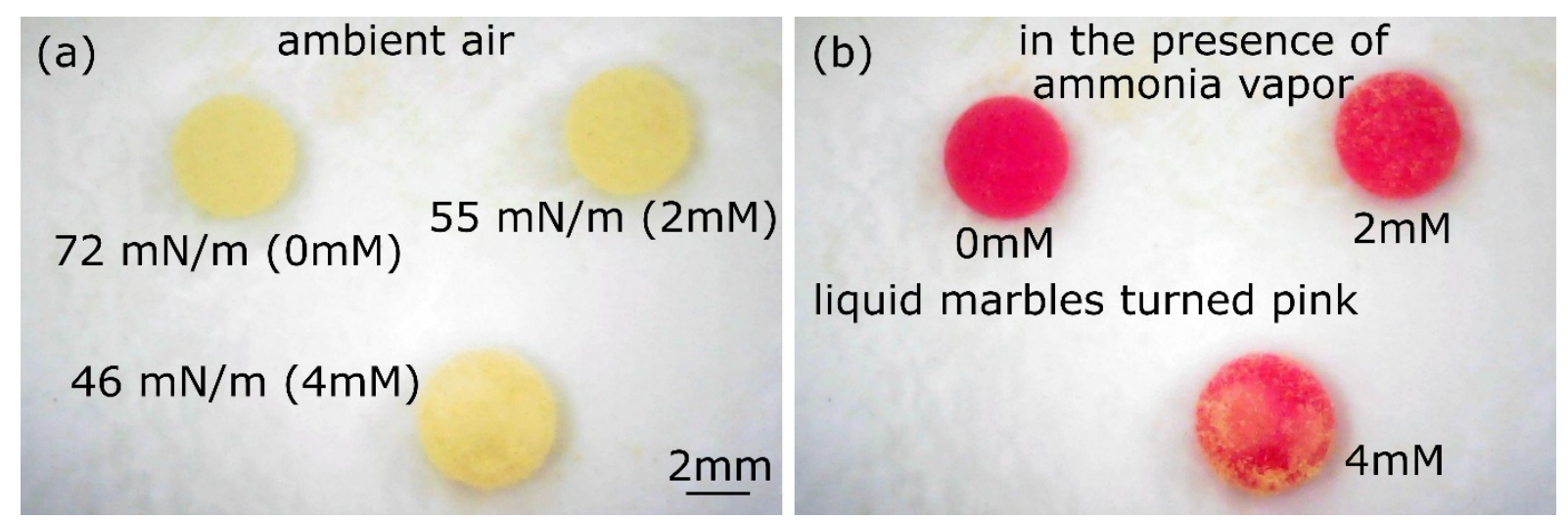

Figure 9. Liquid marbles at surface tension of $72 \mathrm{mN} / \mathrm{m}, 55 \mathrm{mN} / \mathrm{m}$ and $46 \mathrm{mN} / \mathrm{m}$ containing $0.5 \%$ phenolphthalein, a) in ambient air, and b) in the presence of ammonia vapor.

Stability of a liquid marble decreases with the surface tension. ${ }^{[51,67,68]}$ In our experiment, a water liquid marble was highly stable on a clean glass substrate, whereas a liquid marble at a surface tension of $43 \mathrm{mN} / \mathrm{m}$ had lower stability. Liquid marbles at a surface tension of $43 \mathrm{mN} / \mathrm{m}$ collapsed within 70-120s after transferring to the glass substrate (see Supporting Information). The minimum surface tension in our experiment was $43 \mathrm{mN} / \mathrm{m}$ because a lower surface tension would result in an unstable liquid marble which could not be manipulated. Thus, we limited our experimental observations to $43 \mathrm{mN} / \mathrm{m}$.

\section{Conclusions}

We investigated the liquid marble shell and its behavior with different values of the surface tension of core liquid. The shell thickness is independent of the volume. The shell thickness of a $30-\mu 1$ lycopodium liquid marble at a surface tension of $72 \mathrm{mN} / \mathrm{m}$ was $64 \pm 2 \mu \mathrm{m}$, which decreased to $52 \pm 2 \mu \mathrm{m}$ at a surface tension of $54 \mathrm{mN} / \mathrm{m}$. The decrease in the thickness was attributed to the higher degree of particle penetration. Decreasing the surface tension below 


\section{WILEY-VCH}

$54 \mathrm{mN} / \mathrm{m}$ lowered the surface energy of the droplet. As a result, interfacial jamming of the particles occurred at the liquid marble surface, which collects more particles and increases the mass of the encapsulating particles. Eventually, the thickness also increases due to overlapping of the particles. The thickness of the liquid marble increases to $77 \pm 2 \mu \mathrm{m}$ at a surface tension of $43 \mathrm{mN} / \mathrm{m}$. Further decrease of the surface tension prevented the liquid marble from being formed.

Our present work provides new insight towards understanding the particle distribution and how it affects the effective surface tension of a liquid marble. The finding could be useful for studying the stability of liquid marble and its use in sensing applications. Future work on the topic can be extended towards understanding the relationship between the interparticle force and the surface tension.

\section{Supporting Information}

Supporting Information is available from the Wiley Online Library or from the author.

\section{Acknowledgements}

CHO acknowledges funding support from the Australian Research Council (ARC) Discovery Early Career Research Award (DECRA) DE200100119. NTN acknowledges funding support from the ARC Discovery Project DP170100277. PS acknowledges funding support from Griffith University International Postgraduate Research Scholarship and Griffith University Postgraduate Research Scholarship. Authors thank Surasak Kasetsirikul for providing phenolphthalein.

\section{Conflict of Interest}

The authors declare no conflict of interest.

Received: ((will be filled in by the editorial staff))

Revised: ((will be filled in by the editorial staff)) Published online: ((will be filled in by the editorial staff))

\section{References}

[1] P. Aussillous, D. Quéré, Nature 2001, 411, 924. 


\section{WILEY-VCH}

[2] C. H. Ooi, N.-T. Nguyen, Microfluidics and Nanofluidics 2015, 19, 483.

[3] E. Mele, I. S. Bayer, G. Nanni, J. A. Heredia-Guerrero, R. Ruffilli, F. Ayadi, L. Marini, R. Cingolani, A. Athanassiou, Langmuir 2014, 30, 2896.

[4] P. S. Bhosale, M. V. Panchagnula, H. A. Stretz, Applied Physics Letters 2008, 93, 034109.

[5] H. Wu, H. Watanabe, W. Ma, A. Fujimoto, T. Higuchi, K. Uesugi, A. Takeuchi, Y. Suzuki, H. Jinnai, A. Takahara, Langmuir 2013, 29, 14971.

[6] H.-N. Polwaththe-Gallage, C. H. Ooi, J. Jin, E. Sauret, N.-T. Nguyen, Z. Li, Y. Gu, Applied Physics Letters 2019, 114, 043701.

[7] Z. Liu, X. Fu, B. P. Binks, H. C. Shum, Langmuir 2015, 31, 11236.

[8] K. R. Sreejith, C. H. Ooi, D. V. Dao, N.-T. Nguyen, RSC advances 2018, 8, 15436.

[9] C. H. Ooi, E. Bormashenko, A. V. Nguyen, G. M. Evans, D. V. Dao, N.-T. Nguyen, Langmuir 2016, 32, 6097.

[10] M. Dandan, H. Y. Erbil, Langmuir 2009, 25, 8362.

[11] M. D. Doganci, B. U. Sesli, H. Y. Erbil, B. P. Binks, I. E. Salama, Colloids and Surfaces A: Physicochemical and Engineering Aspects 2011, 384, 417.

[12] P. Aussillous, D. Quéré, Proceedings of the Royal Society A: Mathematical, Physical and Engineering Sciences 2006, 462, 973.

[13] G. McHale, M. I. Newton, Soft Matter 2011, 7, 5473.

[14] E. Bormashenko, Current Opinion in Colloid \& Interface Science 2011, 16, 266.

[15] N. M. Oliveira, R. L. Reis, J. F. Mano, Advanced healthcare materials 2017, 6, 1700192.

[16] A. Rendos, N. Alsharif, B. L. Kim, K. A. Brown, Soft Matter 2017, 13, 8903.

[17] D. Zang, Z. Chen, Y. Zhang, K. Lin, X. Geng, B. P. Binks, Soft Matter 2013, 9, 5067.

[18] P. Singha, C. H. Ooi, N.-K. Nguyen, K. R. Sreejith, J. Jin, N.-T. Nguyen, Microfluidics and Nanofluidics 2020, 24, 1. 


\section{WILEY-VCH}

[19] M. Paven, H. Mayama, T. Sekido, H. J. Butt, Y. Nakamura, S. Fujii, Advanced Functional Materials 2016, 26, 3199.

[20] T. T. Y. Tan, A. Ahsan, M. R. Reithofer, S. W. Tay, S. Y. Tan, T. S. A. Hor, J. M. Chin, B. K. J. Chew, X. Wang, Langmuir 2014, 30, 3448.

[21] K. Nakai, H. Nakagawa, K. Kuroda, S. Fujii, Y. Nakamura, S.-i. Yusa, Chemistry Letters 2013, 42, 719.

[22] Y. Chu, F. Liu, L. Qin, Q. Pan, ACS applied materials \& interfaces 2016, 8, 1273.

[23] S.-i. Yusa, M. Morihara, K. Nakai, S. Fujii, Y. Nakamura, A. Maruyama, N. Shimada, Polymer journal 2014, 46, 145.

[24] E. Bormashenko, Y. Bormashenko, R. Grynyov, H. Aharoni, G. Whyman, B. P. Binks, The Journal of Physical Chemistry C 2015, 119, 9910.

[25] C. H. Ooi, A. Van Nguyen, G. M. Evans, O. Gendelman, E. Bormashenko, N.-T. Nguyen, Rsc Advances 2015, 5, 101006.

[26] M. K. Khaw, C. H. Ooi, F. Mohd-Yasin, R. Vadivelu, J. St John, N.-T. Nguyen, Lab on a Chip 2016, 16, 2211.

[27] Y. Zhang, N.-T. Nguyen, Lab on a Chip 2017, 17, 994.

[28] Y. Zhao, Z. Xu, H. Niu, X. Wang, T. Lin, Advanced Functional Materials 2015, 25, 437.

[29] Y. Zhao, J. Fang, H. Wang, X. Wang, T. Lin, Advanced materials 2010, 22, 707.

[30] J. Jin, C. H. Ooi, K. R. Sreejith, J. Zhang, A. V. Nguyen, G. M. Evans, D. V. Dao, N.T. Nguyen, Microfluidics and Nanofluidics 2019, 23, 85.

[31] J. Jin, K. R. Sreejith, C. H. Ooi, D. V. Dao, N.-T. Nguyen, Physical Review Applied $2020,13,014002$.

[32] C. H. Ooi, J. Jin, A. V. Nguyen, G. M. Evans, N.-T. Nguyen, Microfluidics and Nanofluidics 2018, 22, 142.

[33] C. H. Ooi, J. Jin, K. R. Sreejith, A. V. Nguyen, G. M. Evans, N.-T. Nguyen, Lab on a Chip 2018, 18, 3770 . 


\section{WILEY-VCH}

[34] Z. Liu, X. Fu, B. P. Binks, H. C. Shum, Soft Matter 2017, 13, 119.

[35] S. Fujii, S. Sawada, S. Nakayama, M. Kappl, K. Ueno, K. Shitajima, H.-J. Butt, Y. Nakamura, Materials Horizons 2016, 3, 47.

[36] D. Dupin, S. P. Armes, S. Fujii, Journal of the American Chemical Society 2009, 131, 5386.

[37] Z. Xu, Y. Zhao, L. Dai, T. Lin, Particle \& Particle Systems Characterization 2014, 31, 839.

[38] S. Chandan, S. Ramakrishna, K. Sunitha, M. S. Chandran, K. S. Kumar, D. Mathew, Journal of Materials Chemistry A 2017, 5, 22813.

[39] J. Tian, T. Arbatan, X. Li, W. Shen, Chemical Communications 2010, 46, 4734.

[40] J. Tian, T. Arbatan, X. Li, W. Shen, Chemical Engineering Journal 2010, 165, 347.

[41] E. Sato, M. Yuri, S. Fujii, T. Nishiyama, Y. Nakamura, H. Horibe, Chemical Communications 2015, 51, 17241.

[42] N.-K. Nguyen, C. H. Ooi, P. Singha, J. Jin, K. R. Sreejith, H.-P. Phan, N.-T. Nguyen, Processes 2020, 8, 793.

[43] X. Rong, R. Ettelaie, S. V. Lishchuk, H. Cheng, N. Zhao, F. Xiao, F. Cheng, H. Yang, Nature communications 2019, 10, 1 .

[44] R. K. Vadivelu, C. H. Ooi, R.-Q. Yao, J. T. Velasquez, E. Pastrana, J. Diaz-Nido, F. Lim, J. A. Ekberg, N.-T. Nguyen, J. A. St John, Scientific reports 2015, 5, 15083.

[45] R.-E. Avrămescu, M.-V. Ghica, C. Dinu-Pîrvu, D. I. Udeanu, L. Popa, Molecules 2018, $23,1120$.

[46] A. Cassie, S. Baxter, Transactions of the Faraday society 1944, 40, 546.

[47] T. H. Nguyen, K. Hapgood, W. Shen, Chemical Engineering Journal 2010, 162, 396.

[48] E. Bormashenko, R. Pogreb, G. Whyman, A. Musin, Y. Bormashenko, Z. Barkay, Langmuir 2009, 25, 1893. 


\section{WILEY-VCH}

[49] X. Lin, W. Ma, L. Chen, L. Huang, H. Wu, A. Takahara, RSC advances 2019, 9, 34465.

[50] A. Tosun, H. Erbil, Applied surface science 2009, 256, 1278.

[51] C. Wang, Y. He, Colloids and Surfaces A: Physicochemical and Engineering Aspects $2018,558,367$.

[52] P. A. Kralchevsky, K. Nagayama, Langmuir 1994, 10, 23.

[53] P. A. Kralchevsky, K. Nagayama, Advances in colloid and interface science 2000, 85, 145.

[54] E. Bormashenko, G. Whyman, O. Gendelman, Advances in Condensed Matter Physics $2015,2015$.

[55] P. Kralchevsky, V. Paunov, I. Ivanov, K. Nagayama, Journal of Colloid and Interface Science 1992, 151, 79.

[56] P. Gilles de Gennes, P. Brochard, D. Quéré, New York: Springer, 2003.

[57] A. Daerr, A. Mogne, Journal of Open Research Software 2016, 4.

[58] E. Bormashenko, T. Stein, R. Pogreb, D. Aurbach, The Journal of Physical Chemistry C 2009, 113, 5568.

[59] B. P. Binks, A. J. Johnson, J. A. Rodrigues, Soft Matter 2010, 6, 126.

[60] E. Lucassen-Reynders, The Journal of Physical Chemistry 1963, 67, 969.

[61] B. Vonnegut, Review of scientific instruments 1942, 13, 6.

[62] X. Li, H. Shi, Y. Wang, R. Wang, S. Huang, J. Huang, X. Geng, D. Zang, Advanced Materials Interfaces 2018, 5, 1701139.

[63] H. Shi, X. Li, Advanced Materials Interfaces 2020, 2001081.

[64] H.-L. Cheng, S. S. Velankar, Langmuir 2009, 25, 4412.

[65] A. F. Stalder, T. Melchior, M. Müller, D. Sage, T. Blu, M. Unser, Colloids and Surfaces A: Physicochemical and Engineering Aspects 2010, 364, 72. 


\section{WILEY-VCH}

[66] F. Geyer, Y. Asaumi, D. Vollmer, H. J. Butt, Y. Nakamura, S. Fujii, Advanced Functional Materials 2019, 29, 1808826.

[67] E. Bormashenko, A. Musin, G. Whyman, Z. Barkay, A. Starostin, V. Valtsifer, V. Strelnikov, Colloids and Surfaces A: Physicochemical and Engineering Aspects 2013, 425, 15.

[68] P. Singha, S. Swaminathan, A. S. Yadav, S. N. Varanakkottu, Langmuir 2019, 35, 4566.

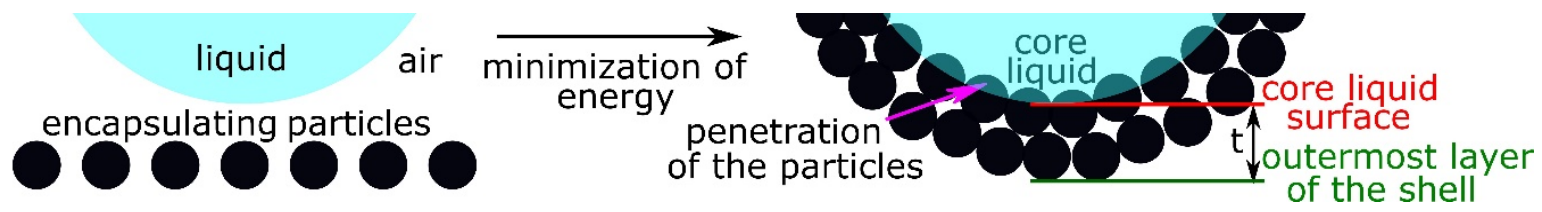

Figure 1. Schematic of the penetration of the encapsulating particles during fabrication, $t$ represents the shell thickness.

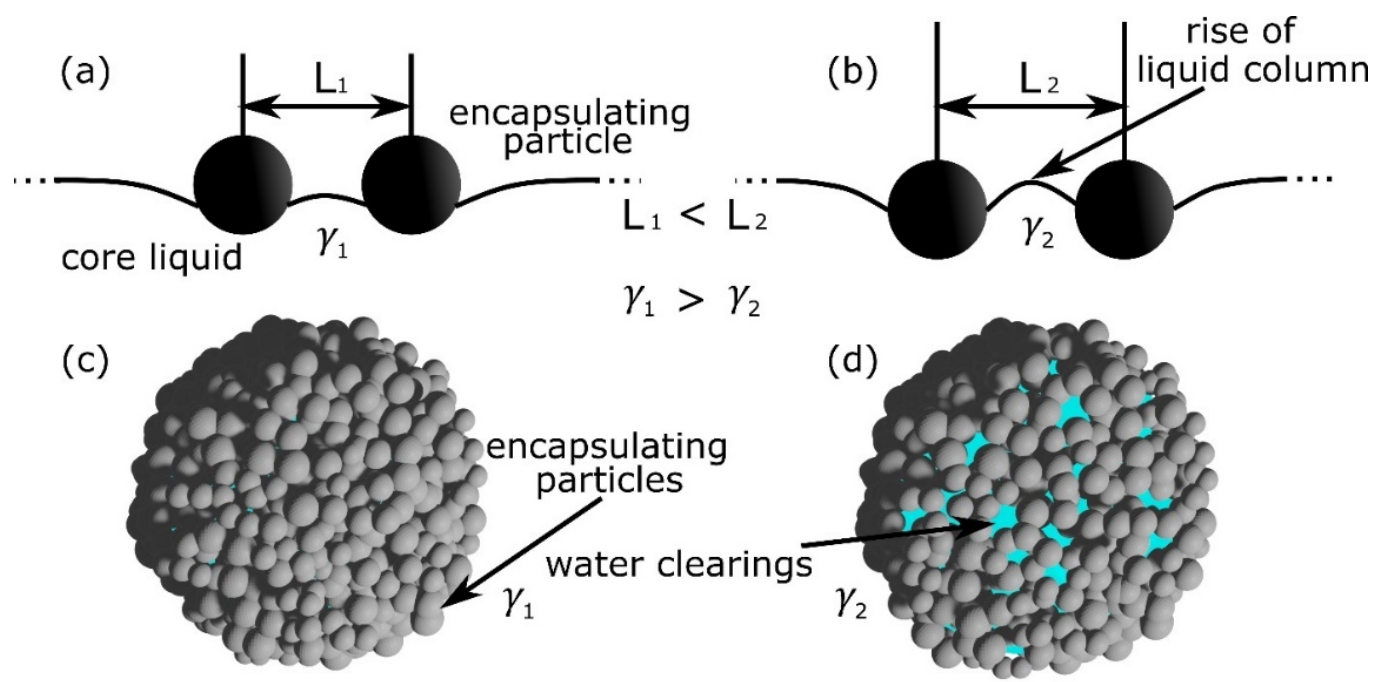

Figure 2. Schematic of the meniscus between two encapsulating particles a) at higher surface tension, and b) at lower surface tension. Depicted particles represent the innermost layer. Schematic three-dimensional illustration of liquid marbles at c) higher surface tension, and d) 


\section{WILEY-VCH}

at lower surface tension. $L$ and $\gamma$ represents the interparticle distance and the surface tension of the core liquid respectively.

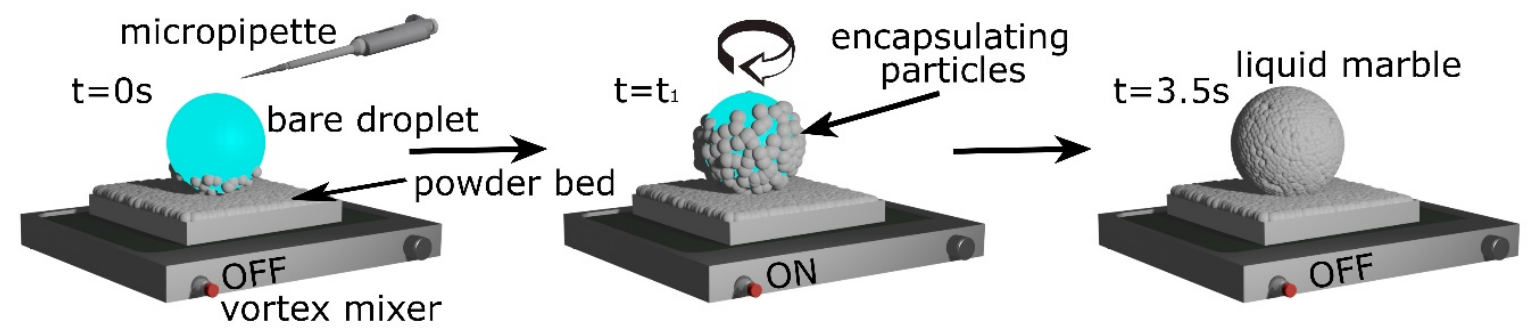

Figure 3. Formation of liquid marbles using a vortex mixer. 


\section{WILEY-VCH}
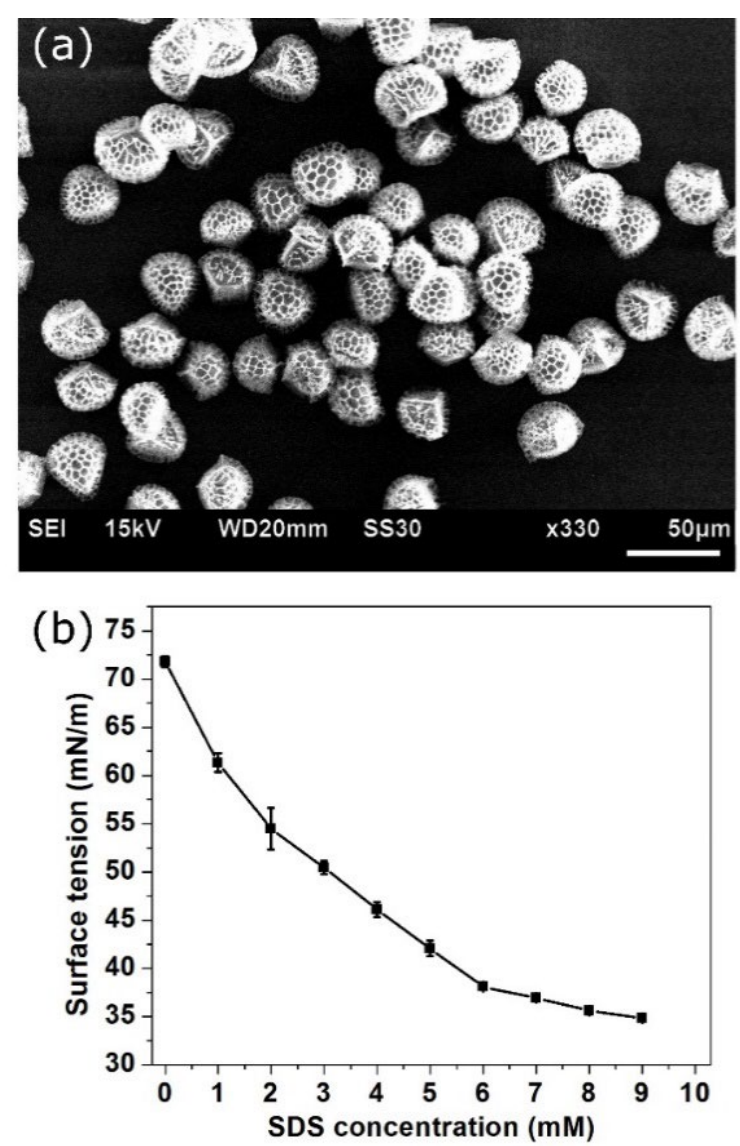

Figure 4. a) Scanning Electron Microscope image of lycopodium powder, b) Surface tension of aqueous SDS solution at $22^{\circ} \mathrm{C}$. 
WILEY-VCH
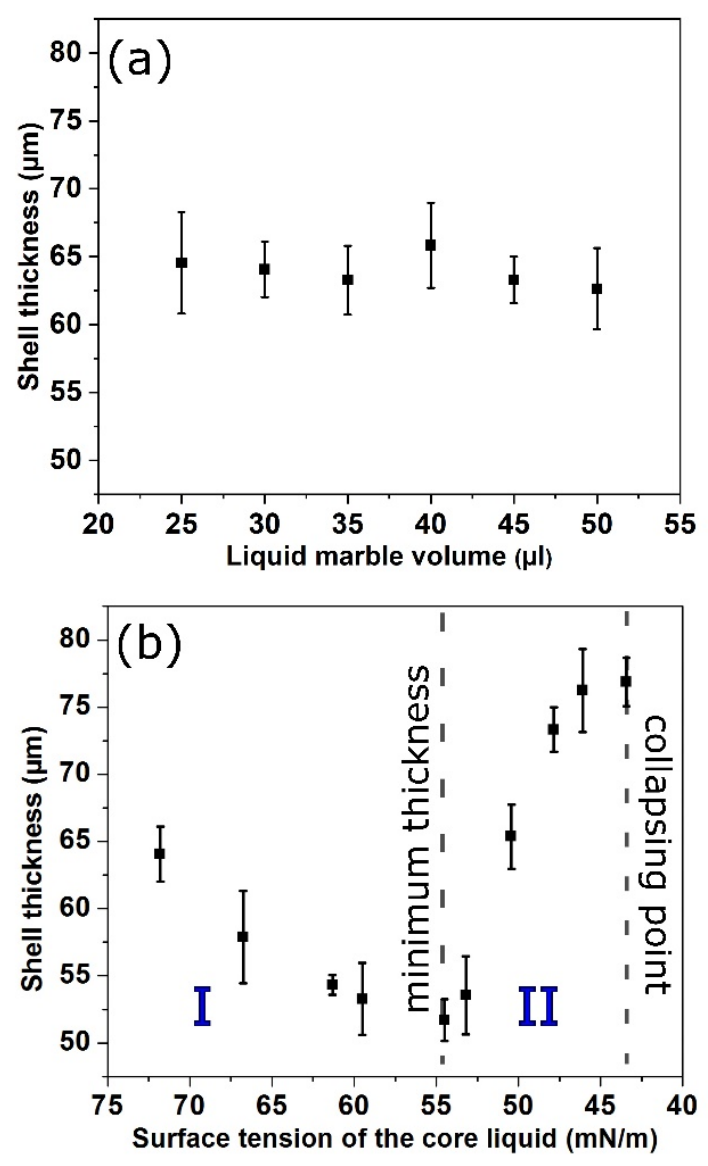

Figure 5. Shell thickness of a) a water-based liquid marble with volumes of $25 \mu 1$ to $50 \mu 1$, and b) a $30-\mu 1$ liquid marble at various surface tension. 
WILEY-VCH
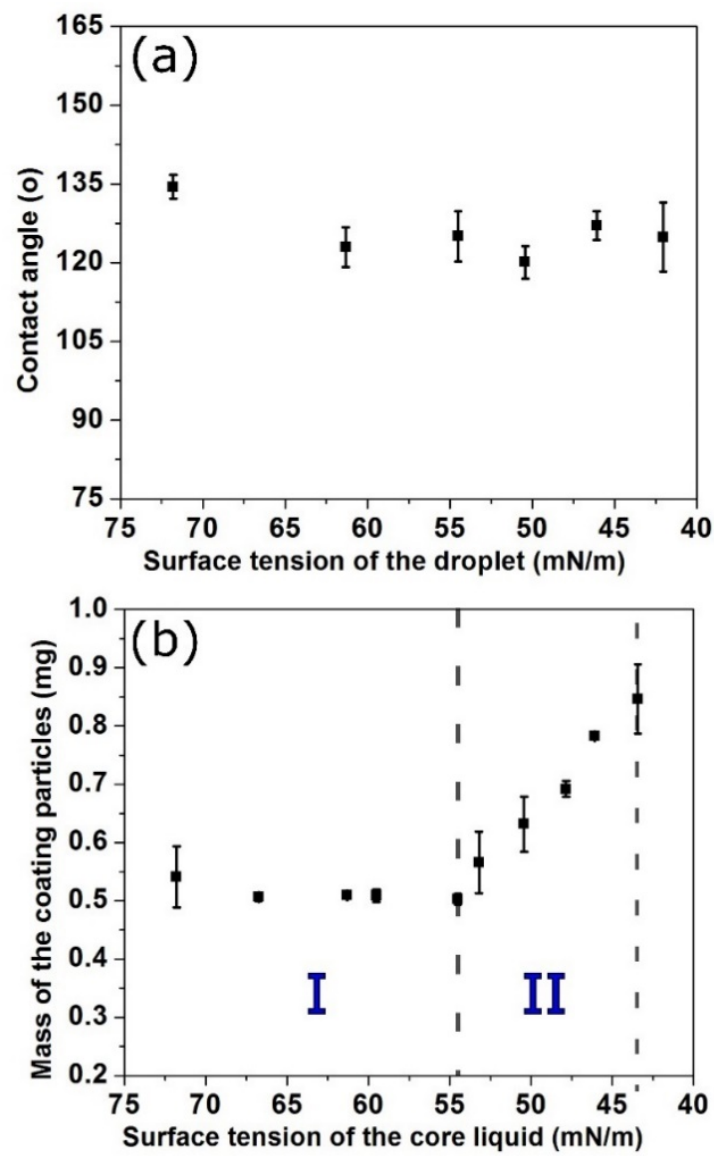

Figure 6. a) SDS concentration versus contact angle, and b) mass of the encapsulating particles of a $30-\mu 1$ liquid marble at various surface tension. 


\section{WILEY-VCH}

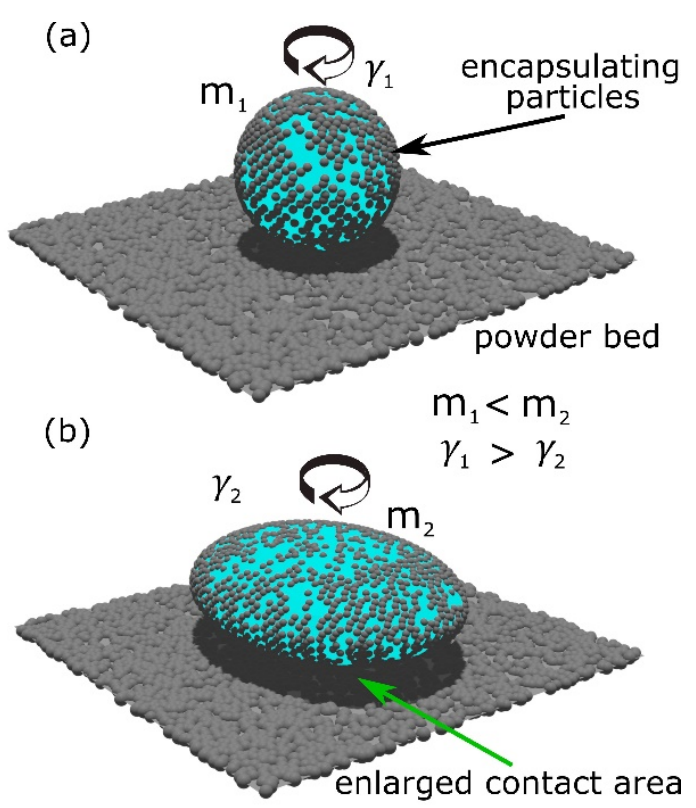

Figure 7. Illustration of the collection of the encapsulating particles through the contact area of a droplet with a) a higher surface tension, and b) a lower surface tension, under the action of vortex mixer, $m$ and $\gamma$ represents the mass of the coating and the surface tension of the core liquid respectively. 


\section{WILEY-VCH}
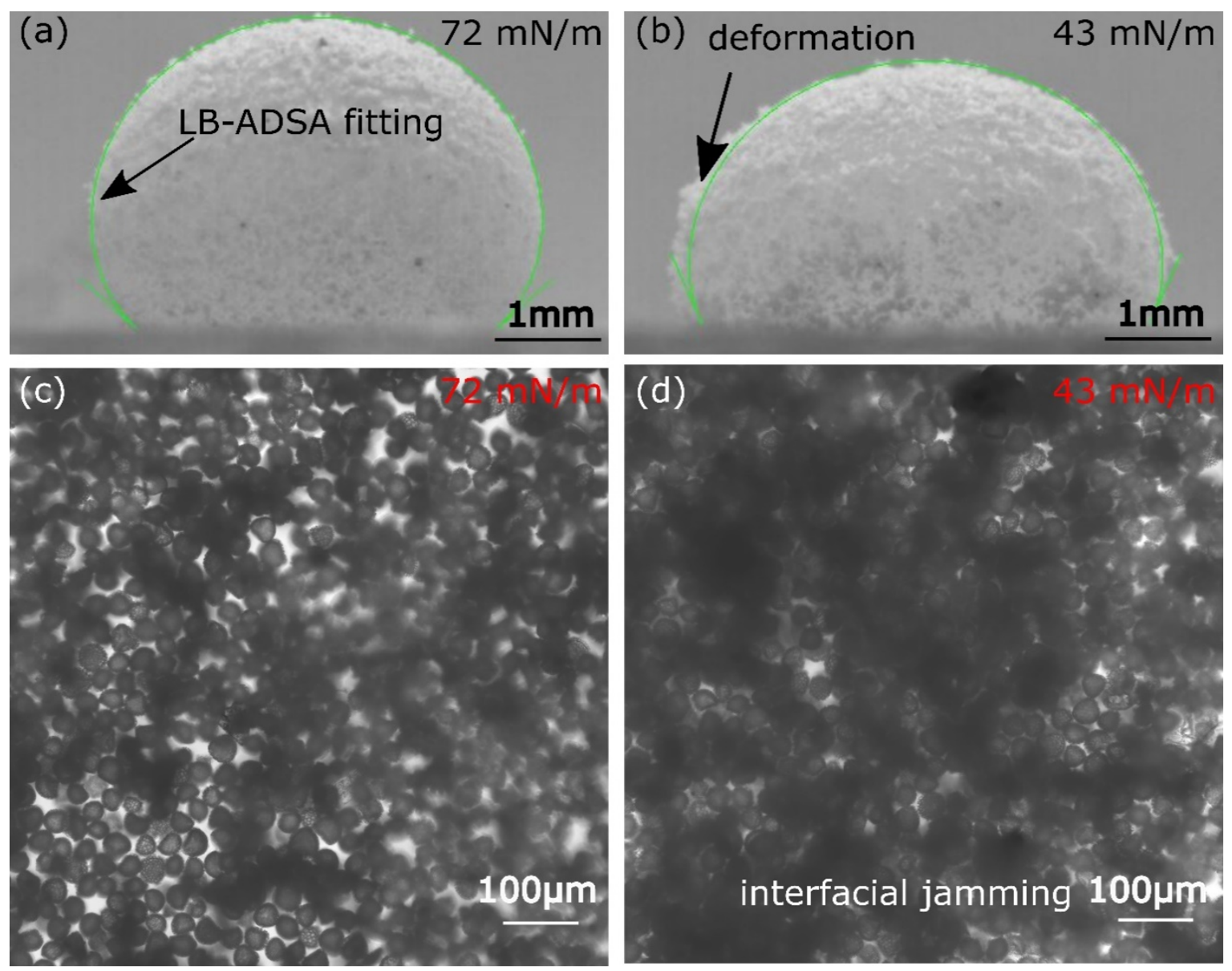

Figure 8. Side view of a liquid marble a) without interfacial jamming, b) with interfacial jamming. Microscopic view of the innermost layer of the liquid marble c) without interfacial jamming, d) with interfacial jamming.

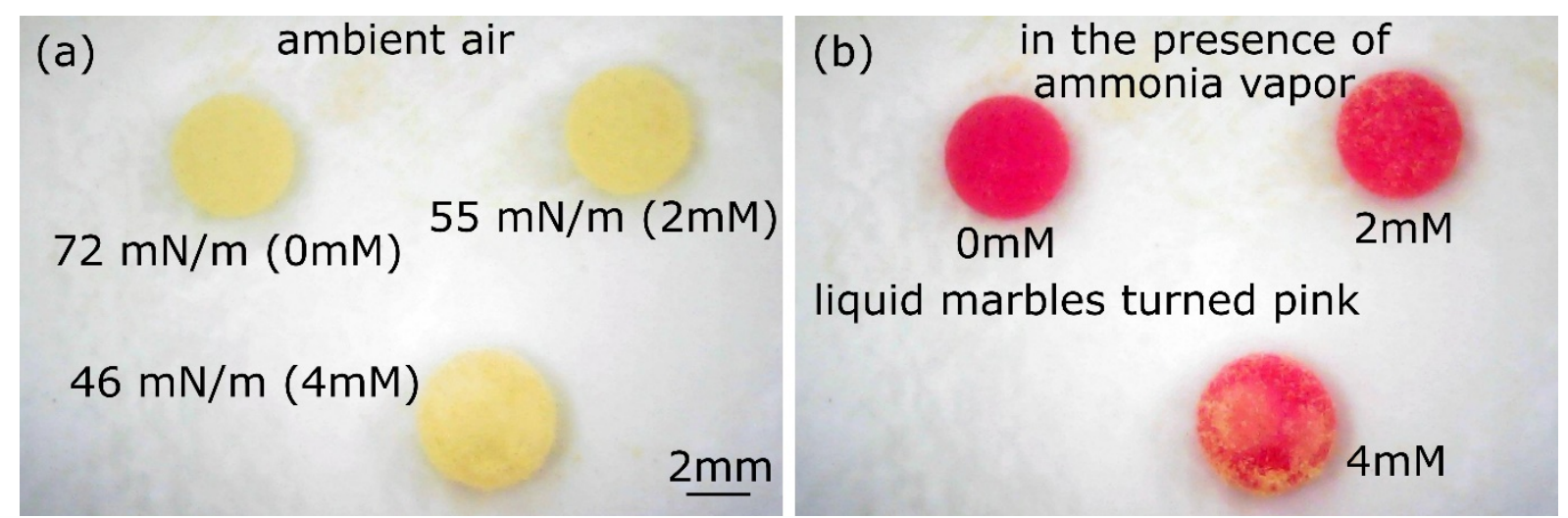

Figure 9. Liquid marbles at surface tension of $72 \mathrm{mN} / \mathrm{m}, 55 \mathrm{mN} / \mathrm{m}$ and $46 \mathrm{mN} / \mathrm{m}$ containing $0.5 \%$ phenolphthalein, a) in ambient air, and b) in the presence of ammonia vapor 
This paper investigates the thickness and the mass of a liquid marble shell at various surface tension values. Surfactant mediated surface tension reduction decreases the thickness, whereas the mass remains almost constant. The trend reverses with further surface tension decrease, where both thickness and mass increase due to interfacial jamming.

Pradip Singha, Nhat-Khuong Nguyen, Kamalalayam Rajan Sreejith, Hongjie An, Nam-Trung Nguyen*, Chin Hong Ooi*

The Effect of Core Liquid Surface Tension on The Liquid Marble Shell

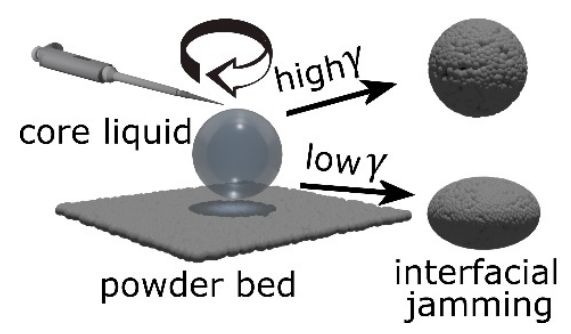

